\title{
Virtual Proving Ground Testing: Deploying Dymola and Modelica to recreate Full Vehicle Proving Ground Testing Procedures
}

\author{
Theodor Ensbury ${ }^{1}$ David Briant ${ }^{2}$ Mike Dempsey ${ }^{2}$ \\ ${ }^{1}$ Claytex USA, Inc., Ann Arbor, Michigan, USA, theodor .ensbury@ claytex.com \\ ${ }^{2}$ Claytex Services Ltd. Edmund House, Rugby Road, Leamington Spa, CV32 6EL, UK \\ \{david.briant, mike.dempsey\}@claytex.com
}

\begin{abstract}
Physical testing of new automobiles is often a lengthy and expensive process. Virtual testing of vehicles offers a much more flexible, efficient solution to testing new vehicles, whilst also providing a more consistent and easier to mange testing environment. This paper presents how the VeSyMA suite of libraries contains the necessary features required to recreate 2 typical physical proving ground tests in the virtual world. Key new features added to the VeSyMA suite to enable this are presented, namely: a new method of defining the proving ground road model using GPS and body accelerometer data, a new driver model capable of conducting a series of scheduled driving tasks (mimicking a human test driver) and new tyre contact models more suitable to typical proving ground rough roads.
\end{abstract}

Keywords: Virtual testing, proving ground, vehicle testing

\section{Introduction}

Development of a robust, high quality product naturally relies upon an extensive program of testing to identify anything which negatively impacts the customer experience. Designed to replicate all elements within a product's usage envelope, such a program seeks, and enables the manufacturer to find, potential issues and validate effective solutions. A program like this can take many forms, including from monitoring actual usage within the field during pre-production; accelerated lifecycle simulation in both deployed or simulated settings; various specific specialist use cases and design limit exceeding, but to name a few.

Automotive product development is no different, complicated by a long, arduous product lifecycle and adherence to various regulatory constraints all over the world. Added complexity arises from the safety-critical nature of an automobile, which further enhances the need for a wide scale testing program to be conducted by the Original Equipment Manufacturer (OEM).

This paper will establish how the Vehicle Systems Modelling and Analysis (VeSyMA) suite of libraries can be deployed to recreate typical real-world testing scenarios in the virtually using Dymola and Modelica, focusing on offline, non Driver-in-the-Loop (DiL) simulation.

\subsection{Standard Automobile testing}

To prepare an automobile for market, testing at various stages of the automobile development process is conducted by an OEM. Some testing will be to fulfil regulatory requirements, with other testing unique to the OEM, often focused on specific areas of interest which the OEM prioritizes. This can include proof-of-concept evaluation. Typically, an OEM test program involves a high amount of physical mileage conducted in prototype, pre-series production and series production vehicles. Various scenarios can be chosen depending on the need, which can involve the vehicle having to be driven extensive amounts of distance or transported around the world to undertake environmental testing. Some testing can be done in laboratory conditions, but non-static full vehicle testing often requires the vehicle to be transported to another part of the world.

This means full vehicle testing can be an expensive and time-consuming endeavour, due not only to the costs associated with producing prototype or pre-series vehicles, but also to the need to transport the vehicles around the globe for testing. Resource time allocation requirements of testing programs can thus impact the product development cycle, often dictating at what point certain test programs can be conducted, rather than deployment at the optimum time during the development cycle.

\subsection{Virtual testing advantages}

Harnessing simulation tools to test virtually can endow multiple benefits. Elimination of the need to produce the same number of prototype and pre-series vehicles is an obvious area cost and time saving to the OEM, in addition to being more environmentally sound. Reducing the amount of travel associated with testing programs further reduces cost, time and environmental expenditure.

Beyond this, virtual testing is also an inherently more flexible process than physical testing. Proof-of-concept testing can be done at a much earlier stage of the design process, whilst durability evaluation can begin to be conducted at earlier stages of the design cycle, as immature designs can be evaluated in the same manner 
as mature designs at the full vehicle level. This leads to the possibility of shortened development schedules, as design issues can be identified earlier and eliminated sooner, with less resources going into failed design elements. Virtual testing can also aid the efficiency and optimisation of components; more inclusive, targeted and varied testing of components to cover more use cases can be conducted during the component development stage.

Virtual testing also offers the possibility of a more consistent testing environment, with greater control of various factors which effect results. Ambient temperature, wind speed and wind direction can all effect physical testing; they can be controlled far more tightly in the virtual environment than in the real world. Human variance when driving the vehicle can also eliminated in the virtual testing environment, as driver models can be used in their place if desired. All this leads to testing conditions being continually repeatable in the virtual world, something unachievable in the physical realm.

\subsection{Virtual testing requirements}

To faithfully recreate a physical real-world testing scenario virtually, the modelling requirements can be broken down into 3 individual categories:

- Vehicle Model

- Road Model

- Driver Model

The vehicle model is termed as the model tasked with recreating the physical vehicle platform's behaviour, including the tyres. A road model accounts for the ground position at the wheel contact point, with a Driver model simulating the control actions applied to the vehicle by the human driver. Complex aerodynamic and thermal interaction with the atmosphere is neglected in this example to reduce the performance load of the simulation.

Logically, a vehicle model deployed in such an application is required to recreate the vehicle behaviour to the satisfaction, and confidence, of the user. In addition, to be able to fully realize the flexibility virtual testing can provide, it follows that it should be built in a modular way with scalable detail. This enables the user to be able to tailor the complexity of the simulation to their desire, effectively isolating specific use cases or effects. Dymola and Modelica's object-oriented nature makes them the obvious choice for this task.

Requirements for a road model are slightly simpler. It should represent the topography of the road surface the vehicle traverses in enough detail to induce the same vehicle response as occurs in the real world. Local and global topical phenomena (rain, dust, dirt or tyre rubber accumulation), which effect the road surface and influence vehicle response behaviour can be included by varying the road mu value (Segers, 2014). Similarly, the driver model utilized must be capable of recreating the same control inputs as conducted in the real-world test.

\section{Vehicle Modelling}

All vehicle, vehicle system models and tyre models described in this chapter form part of the VeSyMA suite of libraries from Claytex.

\subsection{VeSyMA Approach to vehicle modelling}

As outlined in previously (Hammond-Scott and Dempsey, 2018), the VeSyMA suite of libraries from Claytex provides a complete vehicle simulation solution, scalable to specific customer needs and desires. Comprised of the VeSyMA library itself with multiple subject specific expansion libraries, each subject library can either be used in isolation or with extension libraries at both the system model and full vehicle level. Underpinning the VeSyMA suite is the Modelica Standard Library (MSL) and the Vehicle Interfaces Library; the latter defining the standard vehicle level model interfaces, thus enabling each of the VeSyMA subject libraries to interact on a modular level. This is shown in Figure 1.

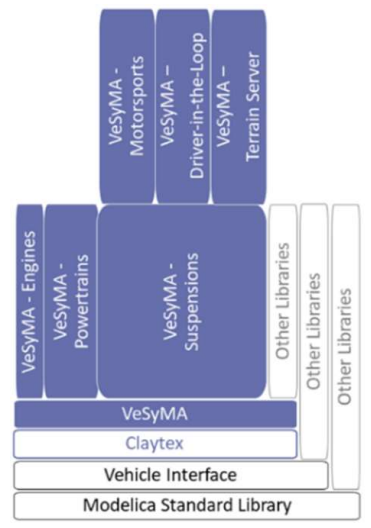

Figure 1. Schematic overview of the VeSyMA suite of simulation libraries.

Acting as a parent library, the VeSyMA library itself defines model templates as well as having the capabilities for straight-line drive cycle testing. Examples of subject specific libraries available for use with the VeSyMA suite include VeSyMA Suspensions, for detailed analysis of automotive vehicle dynamics including 3D multibody suspension, highfidelity tyre models, road and driver models; VeSyMA - Engines, a library devoted to high-fidelity internal combustion engine models; VeSyMA - Powertrains, a library of full 3D multibody driveline and gear train models; VeSyMA - Driver-in-the-Loop, which enables the deployment of vehicle models developed using the VeSyMA - Suspensions (or VeSyMA - Motorsports) 


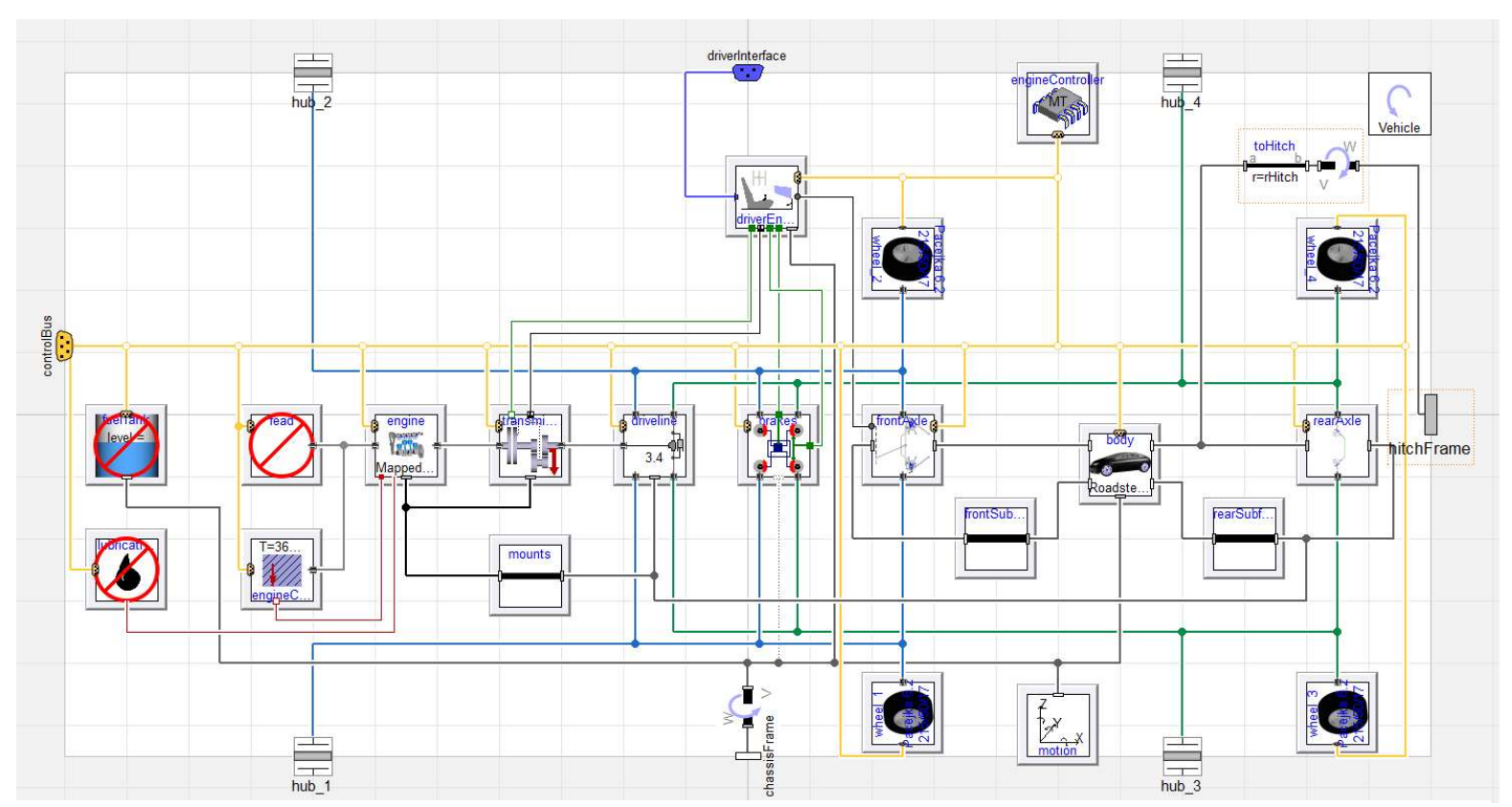

Figure 2. Schematic overview of the RoadsterSportMTRT vehicle model

libraries in DiL real time virtual environments, such as rFPro.

The concept of model reuse is a core principle of the VeSyMA suite. An efficient modelling philosophy, this enables the VeSyMA suite to be a fully scalable, modular solution, where the same models can be deployed in simulations ranging from detailed desktop scenarios, to DiL applications and quick drive cycle evaluations. This enables the user the flexibility to fully utilize several aspects of simulation-based development and investigation over physical testing.

\subsection{Vehicle model}

To demonstrate the capabilities of virtual testing, an example vehicle model was taken from the VeSyMA Suspensions library, namely the RoadsterSportMTRT vehicle model as shown in Figure 2. Being a complete multibody vehicle model, the body is free to move within the virtual environment in all 6 degrees (lateral, longitudinal, heave; roll, pitch and yaw). All subsystem models within this model can be found in either the VeSyMA library or the VeSyMA-Suspensions library.

With a Euro NCAP roadster sport class segment vehicle platform, the RoadsterSportMTRT features a traditional rear-wheel drive layout (RWD) with the drive torque only sent to the rear wheels through an ideal manual transmission model and 1D rotational driveline. No electronic stability modules were included in the vehicle. Lubrication or front engine ancillary drive (FEAD) models were also omitted. A mapped engine model simulated an example internal combustion engine (ICE). More detailed powertrain models could be included by deploying the VeSyMA - Engines or
VeSyMA - Powertrains libraries. Such models are not included as part of the VeSyMA - Suspensions library, thus not featured in this example vehicle model.

Double wishbone suspension was featured at both the front and rear of the vehicle; innovative "aggregate" joints capable of DiL running were utilized. Compliances within the suspension mountings were omitted, as were flexures within the suspension members themselves. No suspension bushing models were used. As the suspension linkages were full multibody models, each link had its own specific mass and inertia properties. Ride spring and damping elements were modelled using translational models, with full multibody anti-roll bar models deployed at both the front and rear axle. Aerodynamic effects were considered, using a simple lift, side force and drag model. All four wheels featured independent brake models, utilizing a controlled elasto-plastic model (Dankowicz, 1999) to model the friction between the disc and the pad. Each brake model featured individual rotating mass models to account for the gyroscopic effects of rotation, along with a separate, static calliper mass model.

The variable step Radau IIa - order 5 stiff solver was used with a tolerance of 0.0001 to simulate the vehicle model during test scenarios. 116 continuous time states were selected when the vehicle model was translated. As this is an generic example vehicle model, it is a qualitative representation of a vehicle using approximate data. Due to this, it enables qualitative judgements to be made regarding it (and surrounding simulation tools), rather than specific quantitative judgements. 


\subsection{Tyre and Contact Modelling}

A key component of any vehicle simulation is the tyre model used. In the example vehicle a fully combined lateral and longitudinal slip Pacejka tyre model was used, corresponding to Magic Formula (MF) 6.2. Behaviour and response were thus non-linear. Included is asymmetric tyre behaviour due conicity and ply-steer with a kelvin spring damper modelling the vertical dynamics of the tyre (Pacejka, 2012). Contact frame information is obtained by interfacing with the road model to gain position, road normal and friction coefficients. These inputs are then used by the tyre model to produce forces and torques that are applied to the suspension and powertrain models.

Typical implementations of the Pacejka model feature only a single point of contact; this limits the model's ability to handle terrain which varies with high frequency, where surface/tyre contact can occur in multiple places on a single tyre.
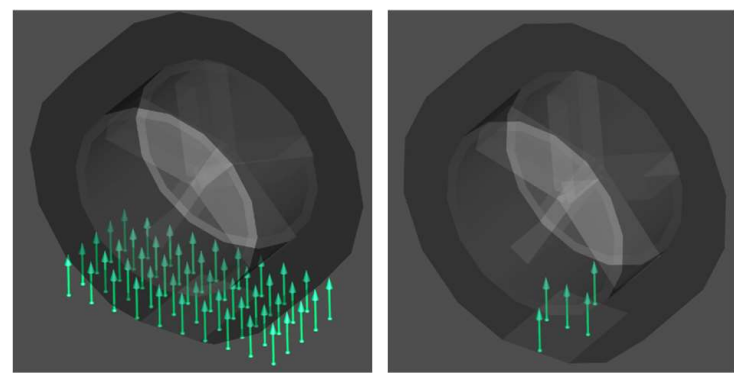

Figure 3. Comparison of multi-point tyre contact models. On the left is an example of the grid contact point model, with the 5 point model on the right.

Tyre model fidelity can be enhanced by deploying a multi-point contact model. Such models interface with a higher frequency or undulating road models, reducing the multiple contact point data down to a single point input for the tyre force model. One outcome is a natural smoothing effect of high frequency/high amplitude road topographical variations. There are 2 implementations of weighted multi-point contact models available in the VeSyMA - Suspensions library, shown in Figure 3; a 5point diamond and a grid pattern. The 5-point contact model features 4 auxiliary outer points arranged in a diamond around a central contact point; spacing of the outer points relative to a central point is controlled by the user by specifying the dimensions of the contact patch. The cross product of the vectors between the central point and the outer points are used to generate 4 normal vectors. And a weighted average of the position of these points is used to produce a contact position. In contrast, the grid pattern contact model sees the contact patch discretized into a rectangular grid of points, aligned with the heading of the wheel hub. The weight of each point is dependent on the penetration depth of the point. The user can define the dimensions and resolution of the grid; each row and each column is equidistant from the next, with the distance ahead being important when considering step changes.

Moving further, the VeSyMA - Suspensions library also supports use of the FTire (Flexible Structure Tyre Model) model, a physics-based tyre model developed by Cosin scientific software. Dymola support is provided by the FTire library, which provides an interface to the FTire external program. The VeSyMA - Suspensions library builds on this interface to integrate into the vehicle models through the road am wheel models only. Unlike mathematical based tyre models, FTire is a fully physical model, which "explains complex tyre phenomena on a strictly mechanical, tribological, and thermodynamic basis". Applicable in short-wavelength, high-frequency studies, such as for vehicle noise, vibration and harshness (NVH), ride comfort studies, load prediction and durability testing, FTire details "temperature, wear, air vibration, (and) rim flexibility" with both rigid and non-rigid road models (Cosin scientific software, 2018).

FTire models require license for use from Cosin scientific software, and thus were not deployed in the use cases presented in this paper.

\section{Road Modelling}

A road model (also termed a ground model), is the model responsible for defining the position of the road surface and commonly includes the driving line information to follow, such as position and velocity targets. Within the VeSyMA libraries there are several different road models, all using the methodology of defining road data relative to the distance along the centreline or driving line, perpendicular offset and road normal height ( $\mathrm{s}, \mathrm{w}$ and $\mathrm{z}$ ). The inner definition of the road at the top level of the experiment is used in conjunction with outer road blocks that can read data from the road using those $\mathrm{s}, \mathrm{w}$ and $\mathrm{z}$ inputs.

The height of the surface of the road is defined by two elements of the road; the underlying topography and the roughness, which is overlaid over the top. Topography of the road defines its general profile (low frequency undulations), which are smooth and considered the general position of the surface. Roughness is a deviation around the topographical position along the road normal to apply higher frequency roughness (bumps) and surface height change events such as kerbs or potholes. Such separation of the two improves efficiency and allows for more accurate measurement relative to the road. For example, during vehicle pitch or roll, the roughness does not influence the measurements taken as the data is measured relative to the smooth underlying surface, improving the accuracy and speed of simulation.

There are several other road types that are available to use in the VeSyMA libraries, which allow for alternative methodology while maintaining the same 
interface, using the road blocks. But FTire uses its own road model which defines the road models and is passes data to the wheels within vehicle model.

\subsection{Defining road topography}

Two approaches can be taken to defining the road model topography; either a real physical road/venue can be recreated, or a fictional road can be used. Both approaches have value depending upon the type of study to be undertaken. For instance, a fictional road can be a road which is impossible to build in real life, like a perfectly flat plane to evaluate spring and damper settings without interference from body forces generated by road topography or roughness. Within the VeSyMA and VeSyMA - Suspensions libraries, there are several functions which enable a road model to be generated from inputted data points. These enable the user to build their own road models of any topography (3D geometry, width, banking angle, friction coefficient and roughness) they wish. In addition, road model building from curvature is also supported, with several functions supporting the development of various specialist roads, such as constant radius circles, slalom courses and figure of eight roads.

It is however often desirable to recreate a physical road for use in virtual testing. One method of doing this is to have the road (or circuit) laser scanned using LiDAR to generate a $3 \mathrm{D}$ point cloud of the surface. Such methods are highly accurate; VeSyMA - Terrain Server provides the tool chain required to interface a VeSyMA suite vehicle model with the full laser scanned driving environments found in rFpro. However, getting a road scanned in such a way is a prohibitively cost intensive endeavour, which may not be suitable. Therefore, the VeSyMA - Suspensions library features a specialist road building function called "RoadFromLoggedData" which builds a road model from GPS and body accelerometer data. This method of building a road model is useful in applications where it is not possible to or simple to laser scan a road (too costly, too time consuming, or the user does not have the permission to undertake such an endeavour) yet the user desires a road model. Furthermore, this method only requires 6 channels of data from 2 sensors, which can often be found on data logging equipment as simple as phone.

As most commercially available GPS units use the Universal Transverse Mercator (UTM) format, the GPS data can easily be converted into $\mathrm{x}, \mathrm{y}$ and $\mathrm{z}$ coordinates by reversing the Gauss-Krüger projection (Kawase, 2013). Based upon dividing the Earth's surface into 60 north/south zones each measuring $6^{\circ}$ longitude, each zone is an effective projection of the curved surface of the Earth onto a flat plane (U.S. Geological Survey, 1997). Transformation parameters utilized are those found in the World Geodetic System 1984 (WGS 84) spheroid model of the Earth (National GeospatialIntelligence Agency, 2005). The only data the user is required to have beyond the GPS sensor output is knowledge of whether the road to be modelled is in the northern or southern hemisphere (a sign change occurs depending on the hemisphere inhabited in the UTM system) and the UTM zone the data was logged in, which can be looked up on a standard map (Kawase, 2013). This is a valid method of detailing road topography, as the Gauss-Krüger projection has been shown to be accurate to "a few nanometers" (Karney, 2011).

Body accelerometer data ( $\mathrm{x}, \mathrm{y}$ and $\mathrm{z}$ axes) is utilized to determine the banking angle of the road, as experienced by the vehicle. For the function to give accurate results, it is advised that the accelerometer data is smoothed sufficiently to present the low frequency fluctuations of data clearly, in addition to being recorded on a vehicle which experiences low roll and pitch angles. Correction for vertical accelerations created by extremely high aerodynamic forces (such as downforce on a prototype racing car) is advised. The following equation is used to determine the banking angle as experienced by the vehicle:

$$
\theta=\operatorname{atan}\left(\frac{G_{\text {Vertical }}-1}{G_{\text {Lateral }}}\right)
$$

This method of deriving the banking angle is considered the standard method when converting logged vehicle data and has been used extensively in motorsport applications (Segers, 2014)

As the aim of the function is to describe the low frequency undulations in road topography, it is advised that the data used to build a road using the "BuildRoadFromLoggedData" function is filtered prior to usage with an appropriate filter and pass band frequency, as well as being logged a non-excessive rate (around 20Hz).

\subsection{Defining road roughness}

As explained earlier in this paper, road roughness is effectively "overlaid" onto the existing road topography, meaning roughness as defined is the deviation of $\mathrm{z}$ height relative to the underlying road topographical data. Therefore, this means that the standard VeSyMA road models require the roughness to be defined independently of the road topography. There are two main ways to do this: implement a standardised friction grade or define a specific roughness table, with data generated from another source.

Each VeSyMA suite standard road model features the ability to deploy an ISO 8608 standard roughness grade; various grades are available, designed to be characteristic of paved road surface quality in different states of wear (Agostinacchio et al, 2013). Available options are:

$$
\begin{aligned}
& \text { - } \quad \text { A (Very Good) } \\
& \text { - B (Good) }
\end{aligned}
$$


- C (Medium)

- $\mathrm{D}(\mathrm{Bad})$

- E (Very Bad)

Defined as a 2D implementation of roughness, each roughness profile is comprised of various excitation frequencies to generate a singular roughness value. Various differing grades provide different amplitudes, whilst the user can determine the range of frequencies which are used to define the roughness in each case. Roughness values generated this way are valid along the heading direction of the road, therefore care should be taken if the vehicle yaws at high angles relative to the road heading direction. Despite this, this method provides a useful method of including generalised roughness values when specific data does not exist.

A user can also specify a roughness table directly into the road when the road model is built using one of the in-built VeSyMA road building functions. Being a 2D table, this enables the user to specify the roughness not only as a function of the road centreline, but also at various road width points perpendicular to the road centreline.

\subsection{Road feature modelling}

Actual physical roads do not solely comprise the driving surface, nor do the vehicle tyres only contact the surface immediately within the contact patch; therefore, there needs to be consideration of road features within the road models.

Such features typically comprise of large protrusions above the road surface, such as kerbs, speed bumps and irregularly large bumps. Features such as these are available to deployed in a cylindrical form, as additions to the road surface. The user can specify their location on the road, as well as their radius, which enables control of their height above the road surface. The multipoint tyre contact models are recommended for use with road kerbs. Specifying a negative height of such a road feature can be done to model depressions in the road surface, such as pot holes.

\subsection{Advanced road formats (VeSyMA - Terrain server, Open CRG, Open Drive)}

Moving beyond road models and features which form part of the standard VeSyMA roads, various advanced options are available for the user to deploy in a VeSyMA vehicle simulation.

Despite the increased cost of production, point cloud data remains a popular method of defining a road in acute detail. Road models made of point cloud data are very high fidelity, with even the smallest details of the surface, surroundings and topography represented. Such formats are popular in DiL applications, such as rFpro, where they are used to recreate various real-world environments, alongside standard rFpro road model definitions. The VeSyMA - Terrain server library provides an interface between the rFpro Terrain Server and the offline Dymola simulation, meaning rFpro point cloud road surfaces (and standard format) can be deployed in VeSyMA vehicle experiments. This enables consistency between the road model used within the DiL and offline simulations. It also allows offline simulations to use high fidelity road models, which exactly match measured road surfaces for improved accuracy of evaluation experiments. Support for all tyre contact methods used in rFpro is also included.

OpenCRG (Curved Regular Grid) format road files are also supported by a specialist road model within VeSyMA, termed the OpenCRGRoad. Growing out of an internal Daimler AG road format in 2008, OpenCRG is a widely used file format for the detailing road surfaces (OpenCRG, 2018). Characterised by a curved reference line with a regular elevation grid, the Open CRG format enables the road topographical data to be accurately and efficiently stored. It defines a specific file structure for this purpose, therefore a specialist road model is used in the VeSyMA suite to support use. (Rauh et al, 2008).

Complimentary to the Open CRG format is OpenDRIVE, a file format for the description of road networks. Another open source format, it is developed and maintained by VIRES Simulationstechnologie $\mathrm{GmbH}$ and designed to enable the user to select routes from larger Open CRG databases (OpenDRIVE, 2018). The VeSyMA - Suspensions library features a tool which creates road files in the VeSyMA road format using a GUI that uses the OpenDRIVE road file to define the route and add velocity targets.

\section{Driver Modelling}

To be able to perform vehicle manoeuvres using conventional vehicle controls, driver models output all demands matching those found in a normal vehicle. Such models can be considered as belonging to one of two categories: Open Loop or Closed Loop, depending on control type. Open Loop drivers do not use inputs from the vehicle or road to produce control demands; they have predetermined outputs, normally dependent on time. Closed Loop drivers use inputs from the vehicle and compare them to targets, either gathered from the road or internally from the driver, producing thus a control demand to achieve those targets.

The interface from the driver to the vehicle uses the same variable naming that is found in the vehicleInterfaces library, which is used as a base, allowing for improved interfacing with other vehicle models.

\subsection{Closed Loop Driver Methodology}

The Closed Loop driver models have a methodology laid out by the template with replaceable components allowing for adjustable methodology. The methodology 
follows in following order and is broken into separate sub-models within the driver, as shown in Figure 4:

1. Senses: Gathers information from vehicle and interprets inputs into usable variables.

2. Planning: Uses interpreted variables to compare against targets.

3. Controllers: Uses the comparisons in the planning to produce a Normalised demand.

This demand is then outputted to the vehicle, where it is received by a driver environment that converts it from normalised demands to real inputs. These inputs, used by the vehicle components, match real measurable values, such as steering wheel angle or pedal position.

The Senses block, alongside routing variables from the vehicle to the driver, also converts position into road and driving coordinates, such that they then can interact with the road to produce target variables.

The planning block is broken down into 3 separate planning areas: longitudinal, lateral and gearbox planning. Each planning block, while predominantly independent, can also be fed by other planning blocks. These blocks take the inputs from either the road and then output achievable targets, such as velocity, that the driver achieves.

The controller blocks, Longitudinal, Lateral and Gearbox, use the targets from the planning block and compare them to the output of the senses block to provide demands.

Both the lateral and longitudinal controllers use modified PID based controllers to produce demands with multiple, separate "Look Ahead" points from the planning block that gather target data ahead of the current vehicle position. For Longitudinal planning that could be target speed, for lateral planning it is driving line position and heading.

To control the driver characteristics, all control variables have been propagated to the driverProfile, a replaceable record, that allows large changes in driver response to be changed with one redeclaration.

Given that there are several different types of vehicle control that a driver would encounter, a controlType selection regarding the gearbox type defines which elements of the driver are active and the types of outputs. This can reference the same variable that exists in the vehicle which further automates the linking of driver to vehicle, requiring less modification of the driver to change between vehicles.

\subsection{Test Drivers}

The Closed Loop driver, described above, uses a consistent method of target and demand generation. However, for a test such a durability cycle, which can contain both speed, gear and other Closed Loop demands and Open Loop, control specific output demands in the same test, this is not enough. An extension to the pre-existing Closed Loop driver is required to include this flexibility. Said extension is required to maintain the same ability to follow Closed Loop targets, as well as specified open and closed demands in any order; all with the ability to return to full Closed Loop mode smoothly.

There are two versions of the test driver extension, the first with a predefined step initiated by a simulation time or driving line position. A Closed Loop demand is swapped for the demand provided by a replaceable source. This is predominantly used for simple tests, where there is only one change in demand. An example of this is the ISO double lane change where the vehicle is to coast through the manoeuvre after arriving at a constant speed.

The second version of the test driver is the test sequence driver, where both the target outputs of the planning block and the demand output from the driver can be controlled. With an expandable number of steps, defined by a record vector, each step contains an activation condition and driver action or target to be followed. Resulting actions or targets must either be output directly as a demand or as a target for the controllers to follow. This, alongside the controlType definition, allows the same driver and test sequence to be built and run with any vehicle. Target or control output is then defined by output from a choice of source.

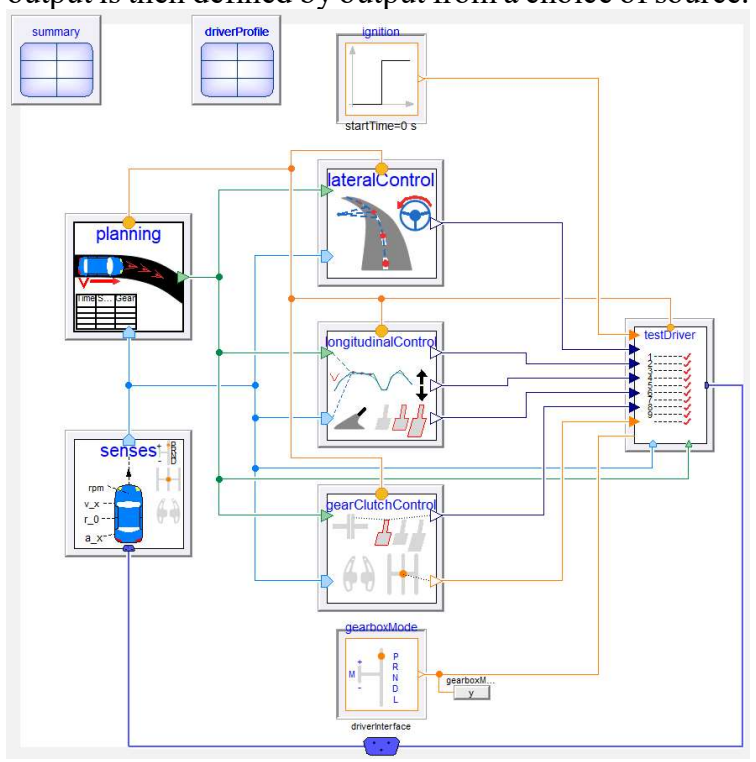

Figure 4. Test Sequence Driver Layout

\subsection{Test Sequence methodology and generation}

The test sequence driver, as mentioned above, uses an expandable record vector with each step defining the condition and output to be used for each step. The activation condition is to be selected from a list of available conditions, which include position, time, velocity and yaw rate. With each condition is the comparison type, either greater or less than or equal to. The driver action, to reduce overheads and complexity, 
is primarily broken down into individual signal-based demand changes. There are also blanket changes to the controls, such as reverting all controls to closed loop. If the driver action has associated targets to define then these are then chosen from a list. If the target, such as a target velocity, is defined then the same closed loop control is used, with the outputs from the planning block being overridden. This therefore retains all the same characteristics as a standard closed loop driver.

During a simulation the driver proceeds through the sequence with the step condition only able to be activated once the previous step is active. This reduces overheads for the driver, with only calculations being active when the current step or awaiting activation.

\section{Example test scenarios}

It has been stated that "Proving grounds are an extremely efficient means of qualifying the durability of vehicle components. They accelerate damage accumulation rates so failures are detectable in a very short period of time" (Halfpenny and Pompetzski, 2011). Therefore, to show the suitability of the VeSyMA suite to simulate proving ground tests, two examples of vehicle durability studies are presented. Specific areas investigated in this paper are high speed laps around a banked circuit and chassis twist/warp bumps, to demonstrate the new additions to the VeSyMA library. High speed lapping of a banked circuit is a standard method of accelerating high-mileage for durability testing, with chassis twist/warp bumps a typical test structural durability, often found at physical proving grounds (Berg, 2015). Each example tests different areas of the vehicle and requires different directions for the driver and vehicle setup. As explained in section 2, due to the vehicle model being a generic example,

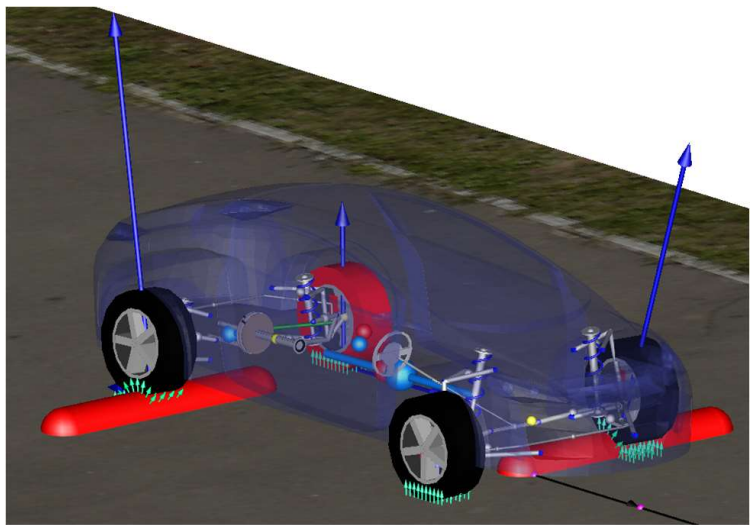

Figure 5. Vehicle rolling over kerbs with wheel loads

results presented here are intended for qualitative analysis rather than specific quantitive analysis.

\subsection{Chassis Twist Bumps}

In the chassis twist experiment there are bumps spaced at roughly a wheelbase length apart on either side of the vehicle. This applies most of the weight of the vehicle onto the opposite corners of vehicles, applying a large amount of torque down the length of the chassis.

For this type of experiment there are several methods of generating road models, as described above. This experiment utilises the VeSyMA - Suspensions road with inbuilt roughness and cylindrical kerbs. The kerbs are spaced equidistantly, roughly a wheelbase length apart on alternating sides of the vehicle, as shown in Figure 5 . The roughness is considered as a medium road surface, using the Grade $\mathrm{C}$ roughness. The contact model used for each of the tyres was the grid contact with 40 contact points each. This allows for the Pacejka

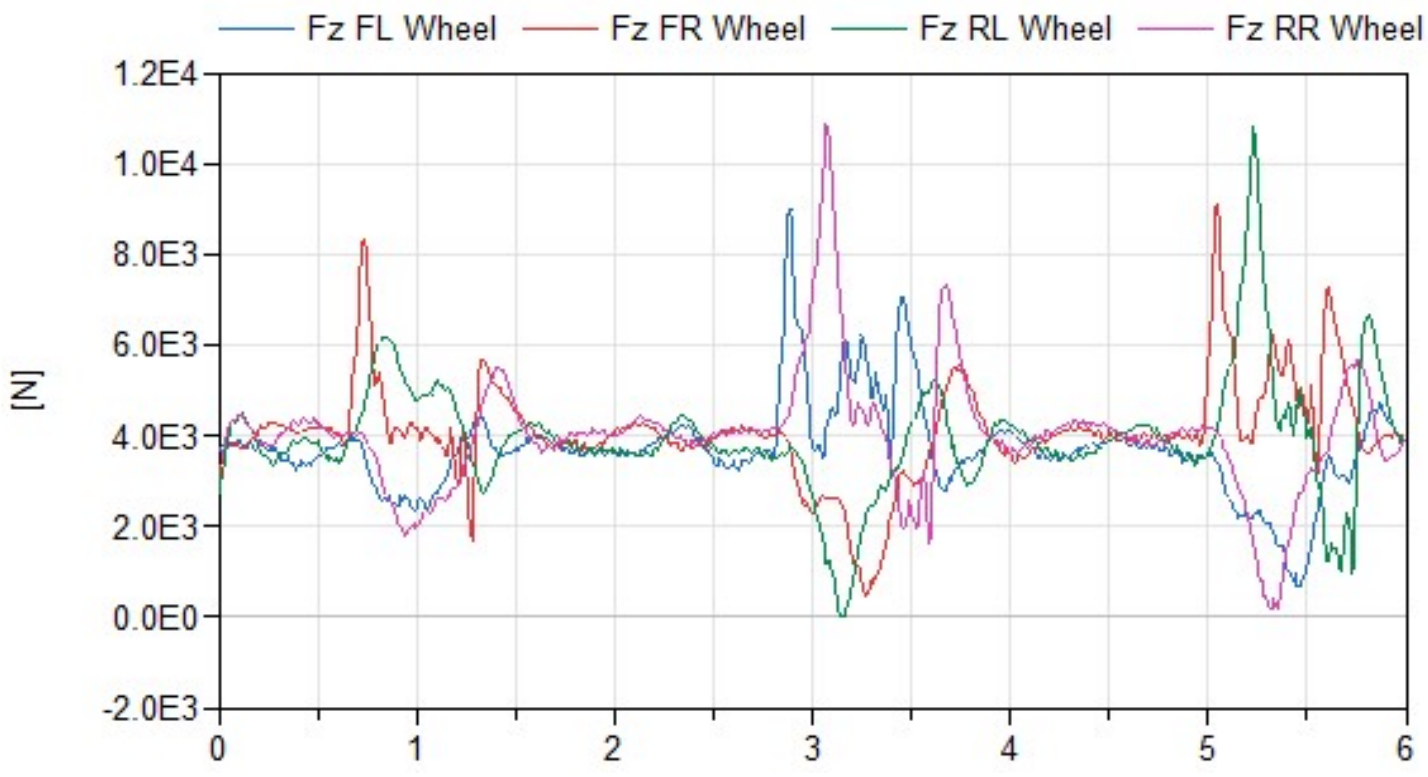

Figure 6. Vertical Wheel Loads against time (seconds) during the chassis twist experiment 


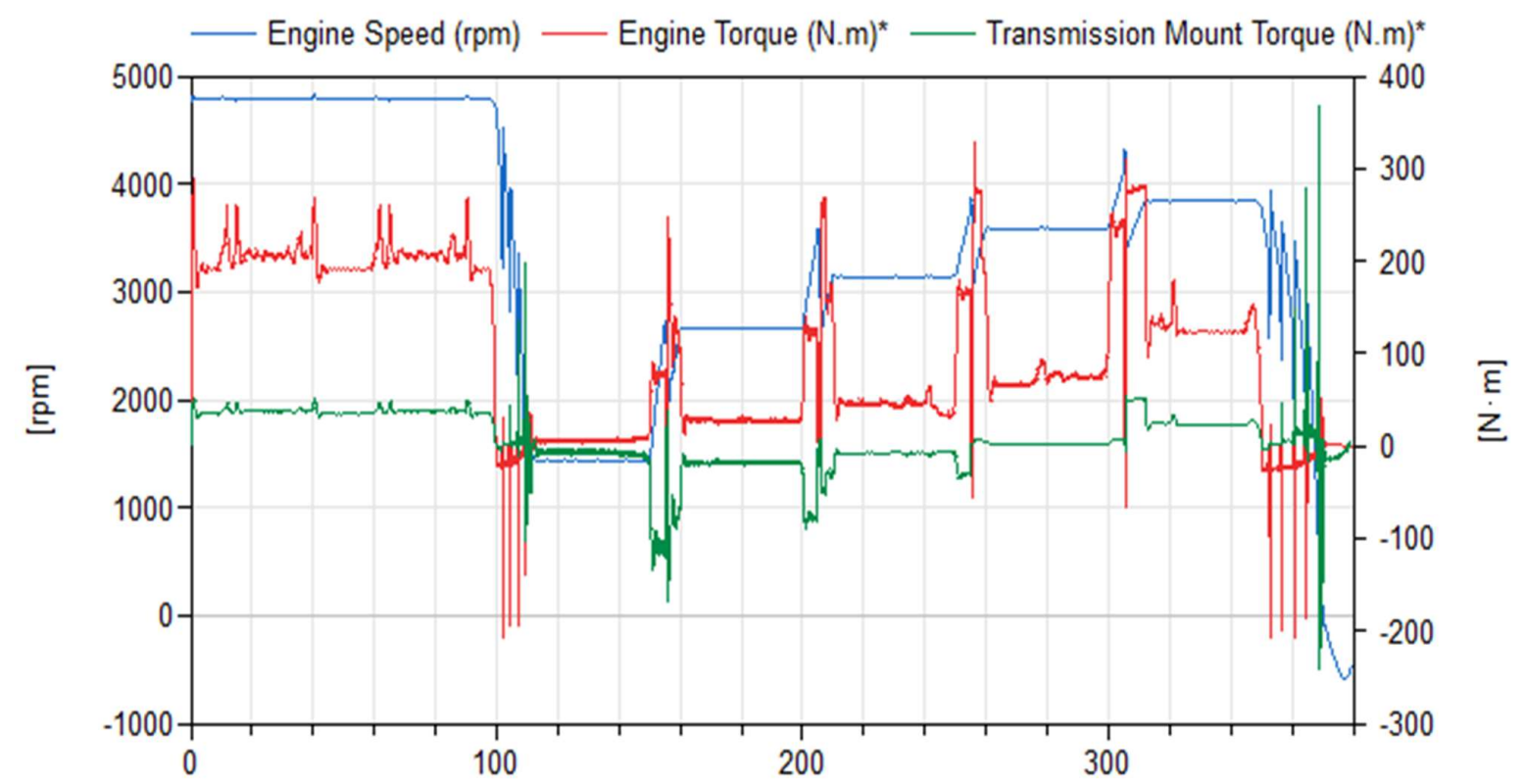

Figure 7. High speed engine speed and Powertrain reaction torques against time (seconds)

model to interact with the kerbs correctly, once more shown by Figure 5 .

The target for the driver is to maintain a constant velocity of $1 \mathrm{~m} / \mathrm{s}$, it holds $1^{\text {st }}$ gear and doesn't use the brake. This allows the vehicle to progress over the kerb at a steady rate without applying brakes such that it rolls over smoothly.

\subsection{High Speed Banked Circuit}

The other test being investigated in this paper is a set of high-speed laps around an oval track with banked corners. In this test the vehicle will be lapping the circuit with both open and closed demands. The roughness will be a smoothest surface Grade A, this matches the road roughness of a refined road track.

This shortened durability cycle includes high-speed laps, high braking sections and incremental speed increases to test elongated driving in different gears. This evaluates drive train durability and is used to gain loads both throughout the drivetrain and reaction forces/torques to the drivetrain mounts.

\subsection{Results}

The chassis twist experiment generated wheel, wishbone, chassis mount and strut top loads that can be used with FEA and fatigue analysis software to generate lift time analysis of both suspension components and the chassis. Figure 6 shows these results, displaying from a qualitive viewpoint the expected wheel load trends, within a 'sensible' force range. As a generic vehicle model was used, the actual specific numerical values of the presented results are not of primary importance. Rather, the trends shown are representative, indicating the simulation is behaving in an accurate manner. With specific vehicle data, this test could be modified to gain kerb strike analysis if the speed was increased to gain a more violent reaction from the wheel input. It can also be extended to move mass around the cabin and include trailers to investigate peak tow bar loads. These results, combined with other, more varied tests can generate a wider understanding of the life time requirements of suspension components. The high-speed durability test (Figure 7) provides additional, higher frequency loads applied to all components, improving the fatigue analysis ability. This test specifically yielded engine reaction and transmission loads applied to the transmission housing mounts at higher frequencies. Changes in the engine and transmission mount torque can be observed as the result of the actions undertaken by the test driver. Conducting such a simulation would provide to the user the expected loads experienced by the vehicle undergoing this test, which could be deployed in more advanced FEA or fatigue analysis software for detailed analysis. Furthermore, they could be used on their own with fatigue metrics to understand how design changes will effect the lifecycle fatigue load the vehicle will endure. Similar caveats regarding the specific numerical value of the results presented in Figure 7 apply as were explained for Figure 6.

\section{Conclusions}

The results presented in this paper demonstrate the capability of the VeSyMA suite by Claytex to provide a virtual testing solution which is capable of recreating proving ground tests, with the introduction of a new driver model, multi point tyre contact models and road modelling features as presented. Furthermore, other methods available to the user through the VeSyMA suite 
are presented, which can be used to include further detail into their analyses.

Example testing scenarios presented show that the vehicle model behaves in a representable manor when travelling at high speed on a banked proving ground circuit, demonstrating the validity of the vehicle and the road model. Multi-point tyre contact models are shown to enable representative rough road and kerb strike experiments to be conducted. The same vehicle model was deployed in both example experiments, albeit with different tyre contact models. This delivers on the assertion of the flexibility of the VeSyMA suite of libraries as a simulation solution, with model reuse enabling the user to focus more on the investigation at hand rather than reformulating simulation models.

\subsection{Further work}

Virtual testing is not just limited to the offline examples demonstrated in this paper. To fully harness the advantages of the simulation solution, it is recommended that such detailed offline work be deployed alongside other forms of simulation, such as DiL and Hardware-in-the-Loop (HiL) testing. Furthermore, as alluded to in the paper, the fidelity of the vehicle model can be improved upon by deploying other subject-specific libraries. Incorporating the VeSyMA - Terrain Server library would enable both detailed offline simulation and DiL testing to be deployed effectively in conjunction with one another.

\section{Related Work}

H. Hammond-Scott and M. Dempsey (2018). Vehicle Systems Modelling and Analysis (VeSyMA) Platform. Proceedings of the 2nd Japanese Modelica Conference, 2018.

M. Dempsey, G. Fish and J. G. Delgado Beltran (2015). High Fidelity Multibody Vehicle Dynamics Models for Driverin-the-Loop Simulators. Proceedings of the 11th Modelica Conference, 2015.

\section{References}

M. Agostinacchio, D. Ciampa and S. Olita (2013). The vibrations induced by surface irregularities in road pavements - a Matlab approach. European Transport Research Review. (2014) 6:267-275. DOI: 10.1007/s 12544-013-0127-8

T. Berg, (2015). Navistar to Use Indiana Proving Grounds For Extensive Testing. Online. Accessed 11 January 2019. Available at:

https://www.truckinginfo.com/129556/navistar-to-useindiana-proving-grounds-for-extensive-testing

Cosin Scentific Software (2018). FTire: Features and Capabilities. Online. Accessed 08 November 2018. Available at: https://www.cosin.eu/products/ftire/

H. Dankowicz (1999). Modelling of dynamic friction phenomena. ZAMM 1999;79:399-409.

A. Halfpenny and M. Pompetzki (2011). Proving Ground Optimization and Damage Correlation. SAE International. DOI: 10.4271/2011-01-0484
H. Hammond-Scott and M. Dempsey (2018). Vehicle Systems Modelling and Analysis (VeSyMA) Platform. Proceedings of the 2nd Japanese Modelica Conference, 2018.

C. F. F. Karney (2011). Transverse Mercator with an accuracy of a few nanometers. SRI International. DOI: 1002:1417

K. Kawase (2013). Concise Derivation of Extensive Coordinate Conversion Formulae in the Gauss-Kruger Projection. Bulletin of the Geospatial Information Authority of Japan. Vol. 60 March, 2013.

National Geospatial-Intelligence Agency (2005). World Geodetic System 1984. Technical Bulletin, United Nations Office for Outer Space Affairs, 2005.

OpenCRG, 2018. Background. Online. Accessed 09 November 2018. Available at: http://www.opencrg.org/project.html

OpenDRIVE, 2018. Background. Online. Accessed 09 November 2018. Available at: http://www.opendrive.org/project.html

Hans B. Pacejka (2012). Tyre and Vehicle Dynamics, $3^{\text {nd }}$ edition. Oxford: Elsevier, pp 356-404.

J. Rauh, H. Schindler, L. Witte, T. Kersten and W. Zipperer (2008). OpenCRG: A unified approach to represent $3 D$ road surface data. AK 6.1.3 Tire Models for Vehicle Dynamics 11.12.2008.

J. Segers (2014). Analysis Techniques for Racecar Data Acquisition, $2^{\text {nd }}$ edition. Warrendale, PA. USA: SAE International.

U.S. Geological Survey (1997). The Universal Transverse Mercator (UTM) Grid. USGS Fact Sheet 149-97. September, 1997. 